\title{
BMJ Open Prognostic factors for post-traumatic stress, anxiety and depression in women after early pregnancy loss: a multi-centre prospective cohort study
}

\author{
Jessica Farren (D) , ${ }^{1,2}$ Maria Jalmbrant (D) , ${ }^{1}$ Nora Falconieri (D) , ${ }^{3}$ \\ Nicola Mitchell-Jones (D) , ${ }^{1,4}$ Shabnam Bobdiwala (D) , ${ }^{1}$ Maya Al-Memar (D) , ${ }^{1}$
} Nina Parker (D) , ${ }^{1}$ Ben Van Calster (D) , ${ }^{3,5}$ Dirk Timmerman (D) , ${ }^{3,6}$ Tom Bourne (D) ${ }^{1,3,7}$

To cite: Farren J, Jalmbrant M, Falconieri N, et al. Prognostic factors for post-traumatic stress, anxiety and depression in women after early pregnancy loss: a multi-centre prospective cohort study. BMJ Open 2022;12:e054490. doi:10.1136/ bmjopen-2021-054490

- Prepublication history and additional supplemental material for this paper are available online. To view these files, please visit the journal online (http://dx.doi.org/10.1136/ bmjopen-2021-054490).

Received 14 June 2021 Accepted 27 January 2022

Check for updates

(C) Author(s) (or their employer(s)) 2022. Re-use permitted under CC BY-NC. No commercial re-use. See rights and permissions. Published by BMJ.

For numbered affiliations see end of article.

Correspondence to Professor Tom Bourne; t.bourne@imperial.ac.uk

\section{ABSTRACT}

Objectives To investigate prognostic factors for anxiety, depression and post-traumatic stress (PTS) symptoms 1 month after early pregnancy loss (EPL).

Design A prospective cohort study. Consecutive women were recruited, and demographic and clinical data were collected. Surveys containing the Hospital Anxiety and Depression Scale (HADS) and Post-traumatic Stress Diagnostic Scale (PDS) were emailed 1 month after a Ioss. Univariable logistic regression was performed to link factors with caseness of anxiety, depression or PTS according to screening measures.

Setting Early pregnancy units of three central London hospitals.

Participants 737/1116 eligible women with an EPL were recruited. 492 responded to HADS and 487 to PDS.

Primary and secondary outcome measures Primary outcome is the area under the curve (AUC) to predict any psychological morbidity (defined as moderate/severe anxiety or depression, or meeting screening criteria for PTS) for each variable. Further outcomes are explained variation $\left(R^{2}\right)$ and $p$ value for any morbidity, and AUC, explained variation, and $p$ value for each morbidity separately.

Results Women who had a past diagnosis of a psychiatric condition were more likely to meet criteria for anxiety, depression or PTS (75\% for current diagnosis vs $55 \%$ for past vs $30 \%$ for no diagnosis; AUC 0.61 ; $R^{2} 8.4 \%$; $\mathrm{p}<0.0001)$, as were those with previous pregnancy loss (48\% vs $30 \%$; AUC $0.59 ; R^{2} 4.3 \%$; $p<0.0001$ ). Most of the assessed factors did not demonstrate potential utility in predicting psychological distress, including gestationa age, overnight admission, time taken for diagnosis, preexisting children and the diagnosis itself (miscarriage vs ectopic vs other) (AUCs $\leq 0.54 ; R^{2} \leq 0.9 \%$ ).

Conclusions Women with a history of mental health problems, or those with previous losses, may be at higher risk of psychological illness 1 month after pregnancy loss. However, prognostic ability was poor overall. All women should be considered at risk.

\section{INTRODUCTION}

Evidence to date has confirmed that early pregnancy losses (EPLs) may be associated
Strengths and limitations of this study

- We have involved a large cohort of women to explore a wide variety of prognostic factors for psychological morbidity after early pregnancy loss (EPL).

- We included women with miscarriage, ectopic pregnancy and resolved pregnancy of unknown location: few studies have included groups other than miscarriage.

- We have assessed for a relationship with anxiety, depression and post-traumatic stress (PTS), both as a combined outcome and individually. PTS has been found to be the most common response after EPL, but has been little studied.

- A weakness is in the use of screening questionnaires for psychological morbidity.

- A further weakness is the drop out of participants: $67 \%$ of those recruited responded to the questionnaire.

with a high likelihood of anxiety, depression and post-traumatic stress (PTS) ${ }^{1-3}$ Given the high frequency of EPLs, and their impact at an important time in a woman's life (at work and at home), it is imperative that focus is given to ways to prevent or treat this psychological morbidity.

A Cochrane review, published in 2012, suggested that there was no evidence, from a total of 1001 participants across six studies, to support offering counselling in various formats to all women following EPL. ${ }^{4}$ However, the predictive validity of those studies was deflated by a floor effect: they included all women with EPL, rather than selecting women who were clinically distressed prior to the intervention and who therefore realistically could show improvement. It is probable that better results could be obtained by targeting treatment towards those who experience clinically significant 
symptoms of distress. Understanding what (if any) factors in a woman's history or clinical encounter are associated with an increased risk of psychological morbidity might enable treatment to be targeted at those at high risk, with better results.

A number of possible prognostic factors have been suggested by previous research, including childlessness, ${ }^{56}$ previous losses, ${ }^{36}$ previous subfertility, ${ }^{7}$ in vitro fertilisation (IVF) pregnancy, ${ }^{8}$ psychiatric history ${ }^{29-11}$ and longer gestation. ${ }^{3}{ }^{12}$ However, many of these have been identified on the basis of retrospective exploratory analyses for statistically significant differences between groups, and the degree to which they may actually be able to explain the variation in psychological morbidity between individuals remains obscure. Furthermore, limited research has been done linking potential factors to PTS symptoms, which, according to this group's recent study, is the most common psychological response. ${ }^{1}$

This explorative study aimed to assess whether, in a large cohort, a prospectively chosen set of potential factors could be used to reliably and usefully predict those with psychiatric morbidity. It is a study to assess for prognostic factors, and therefore without assessment of causation or analysis for confounders.

\section{METHODS}

This is the third report from the Psychological Impact of Early Pregnancy Events prospective cohort study. The first reported on anxiety, depression and PTS at 1, 3 and 9 months in women directly experiencing a loss and a control group in healthy pregnancy. ${ }^{1}$ The second reported on these symptoms in both women and their partners in a cohort of couples. ${ }^{13}$ This report focuses on exploring prognostic factors for morbidity reported at 1 month.

Women with pregnancy losses before 20 weeks (miscarriage (including molar pregnancy), ectopic pregnancy (EP), and resolved pregnancy of unknown location) were recruited from the Early Pregnancy Assessment Units at three hospitals in central London (Queen Charlotte's and Chelsea, St Mary's, and Chelsea and Westminster Hospitals) between 13 November 2013 and 15 March 2016. Exclusion criteria were: age of participant $<18$ years, lack of proficiency in the English language (insufficient, based on the subjective assessment by the researcher, to complete the questionnaire without help or translation), inability to give informed consent, review following voluntary termination of a pregnancy, or if they were already a participant in the study following a previous loss.

Women were recruited consecutively, and could be recruited on the day of diagnosis of a loss or at follow-up (within 1 month of diagnosis) thereafter. Written consent was required. The target sample size of 721 women with EPL was based on data from our pilot study, with the aim to assess for a $20 \%$ difference in PTS prevalence in those with IVF and without, taking into account a predicted
$60 \%$ response rate at 1 month (with the aim to include 440 responders). ${ }^{14}$

The clinical care of women was unaltered by participation in the study. Those with a diagnosis of incomplete or missed miscarriage were offered the clinically appropriate options out of expectant, medical (misoprostol administered by the patient at home) or surgical (under general anaesthesia) management. Women with EP were offered expectant management, methotrexate or surgical intervention (usually laparoscopic salpingectomy) depending on symptoms and clinical markers. Women with resolving pregnancy of unknown location (PUL) were asked to check for a negative urine pregnancy test after 2 weeks. Women with a confirmed diagnosis of a molar pregnancy were referred to the regional trophoblastic centre.

Details of the encounter were prospectively collected, including, for the purposes of this analysis, the woman's age at diagnosis, the date of last menstrual period, the final diagnosis (miscarriage, ectopic, other (PUL and molar)), the dates and outcomes of any scans (including whether a fetal heart had previously been visible in women who were subsequently diagnosed with miscarriage) and number of nights admission. The length of time from the first scan to a diagnosis of loss was calculated. Management was also recorded: if multiple interventions were required (most commonly medical or expectant management followed by surgical), then the final definitive management was used. Record was made as to whether the pregnancy was conceived via IVF.

Women were sent a link to a confidential online survey (in which they were identified by a study number) by email 1, 3 and 9 months after diagnosis of their loss. Only data from the 1 month questionnaire was included in this analysis. Reminders that they were free to withdraw from the study were included in every communication. Without active withdrawal, two reminder emails at weekly intervals were sent to those who did not respond.

As part of the first questionnaire, respondents were asked their ethnicity, their past educational attainment, whether they had experienced past losses, past terminations of pregnancy or had existing children. They were asked whether they had previously been diagnosed and/or received treatment for a psychiatric condition (currently, in the past or no). They were also asked how long they had been trying to conceive. The methods by which these data were obtained, and the groupings used in both data collection and analysis are summarised in online supplemental table 1.

Surveys included two psychometric screening questionnaires: the Hospital Anxiety and Depression Scale (HADS) ) and the Post-traumatic Diagnostic Scale (PDS). Both have previously been used in the pregnancy loss population, and have been shown in multiple contexts to have good psychometric properties. ${ }^{15}$ Further discussion of these measures is included in our primary analysis. ${ }^{1}$ A woman was considered to meet criteria for anxiety or depression if their score fell within the moderate or severe range $(\geq 11 / 21$ for each). For PTS, a PDS score 
Approached to participate $n=1201$

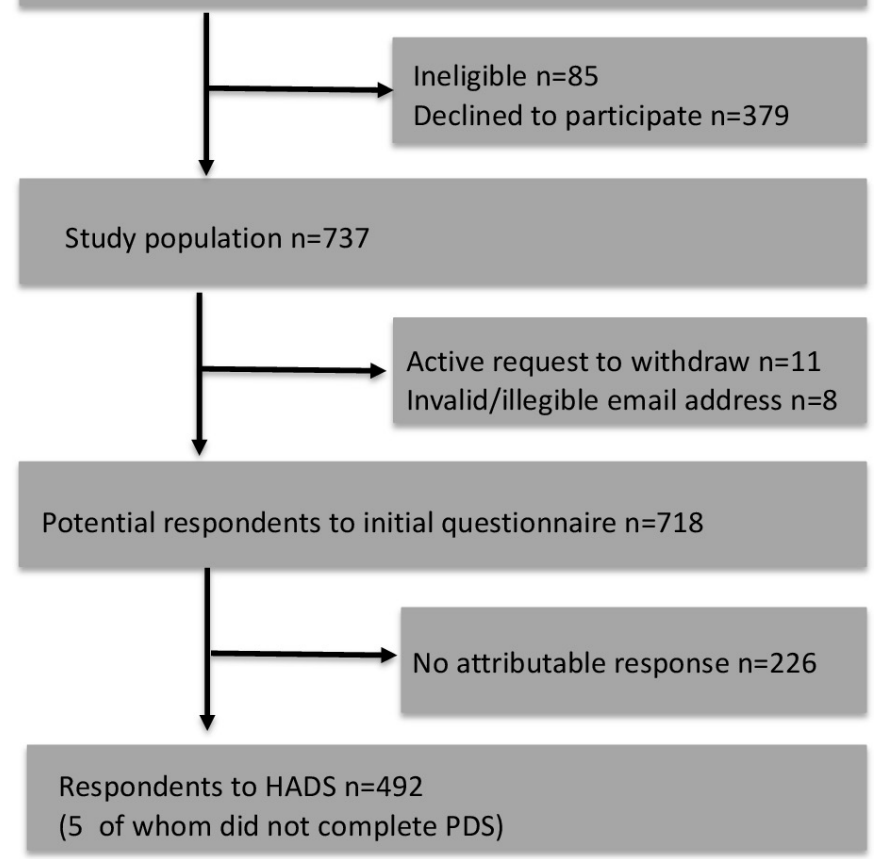

Figure 1 Flowchart of women approached, who agreed to participation, and who responded to the questionnaire. HADS, Hospital Anxiety and Depression Scale; PDS, Posttraumatic Diagnostic Scale.

$\geq 18 / 51$, along with endorsement of the required number of symptoms within each cluster (re-experiencing, avoidance and hyper-arousal), was required. ${ }^{16}$

Exploration of the potential prognostic value of each factor was performed by univariable logistic regression, initially for any morbidity (defined as moderate/severe anxiety or moderate/severe depression or PTS), and then for each morbidity individually. The primary outcome was the area under the receiver operating characteristic curve (AUC) for any morbidity. Further outcomes were the Nagelkerke $\mathrm{R}^{2}$ to quantify the explained variation in the outcome and the likelihood ratio $\mathrm{p}$ value for any morbidity, and the AUC, $\mathrm{R}^{2}$ and $\mathrm{p}$ value for each morbidity separately. Because missing values were limited among responders at 1 month, individuals with a missing value were excluded from the analyses involving that predictor only. The goal was to explore which prognostic factors could be subject to further research for developing a multivariable prediction model.

All statistical analyses were performed using R V.3.6.1. The reporting was based on the Reporting Recommendations for Tumour Marker Prognostic Studies (REMARK) and Strengthening the Reporting of Observational Studies in Epidemiology (STROBE) guidelines.

\section{RESULTS}

A flowchart of women approached, eligible, recruited and who responded is shown in figure 1 . Of the 737 women with EPL who were recruited, 492 responded to the HADS questionnaire and 487 to the PDS. The questionnaires were sent 1 month after diagnosis, and responses were a mean of 40 days after diagnosis (SD, 12; IQR, 32-45). Of those responding to HADS, 366 cases were miscarriage, 75 were EP and 51 were other diagnoses (including resolved pregnancy of unknown location and molar pregnancy). Demographic, background clinical and response data on all respondents is shown in table 1 . There was a small proportion of missing data for all variables except gestational age, for which 84 cases were omitted where this was unknown.

The variable with the highest AUC was past or current diagnosis of a psychiatric disorder (AUC 0.61, $\mathrm{R}^{2} 8.4 \%$, $\mathrm{p}<0.0001$ ) (table 2). Seventy-five per cent $(15 / 20)$ of those with a self-reported current diagnosis of a psychiatric disorder met criteria for anxiety, depression or PTS, compared with $55 \%(45 / 82)$ of those with a past diagnosis, and $30 \%(115 / 382)$ in those without a psychiatric history (figure 2, table 3). AUCs for each morbidity separately were 0.60 (anxiety), 0.64 (depression) and 0.61 (PTS) (online supplemental table 2).

Those with past losses also appear to be at higher risk of any morbidity (AUC 0.59, $\mathrm{R}^{2} 4.3 \%, \mathrm{p}<0.0001$ ): $48 \%$ $(86 / 180)$ women with any previous loss met criteria for any disorder, compared with $30 \%$ (92/307) in the group without previous losses. AUCs for each morbidity separately were 0.59 (anxiety), 0.64 (depression) and 0.57 (PTS).

There is a modest suggestion of prognostic value for time to conceive (AUC $0.56, \mathrm{R}^{2} 2.2 \%, \mathrm{p} 0.02$ ) and ethnicity (AUC 0.57, R 2.4\%, p 0.07). Forty-nine per cent (40/81) women who had taken more than 1 year to conceive met criteria for any disorder, compared with 35\% (105/296) in those taking <1 year, and $30 \%(33 / 110)$ in those in whom the pregnancy was unplanned. Forty per cent (72/182) women of white British ethnicity, 35\% (17/48) of Asian ethnicity, $34 \%$ (17/50) of black ethnicity, $29 \%$ (46/156) of other white ethnicities and 51\% (26/51) of any other ethnicities met criteria for any morbidity.

Factors with little to no evidence of potential prognostic value (AUC $\left.\leq 0.54, \mathrm{R}^{2} \leq 0.9 \%, \mathrm{p} \geq 0.14\right)$ : include: the diagnosis itself (miscarriage vs ectopic vs other), the final maagement strategy, having seen the fetal heart on previous ultrasound imaging (miscarriage only), the woman's age, the woman's educational attainment, overnight admission, previous termination of pregnancy, previous children, IVF conception, duration of the time to diagnosis from first scan, and gestation at time of diagnosis (in those for whom this is known).

For each prognostic variable, AUC, Nagelkerke $\mathrm{R}^{2}$ and $\mathrm{p}$ values for each morbidity separately (anxiety, depression and PTS) were generally similar: there was no suggestion of value for certain psychological diagnoses (online supplemental table 2).

Overall, even those factors likely to be associated with any morbidity (based on $\mathrm{p}$ values) do not seem to have strong prognostic ability (based on the AUC and $\mathrm{R}^{2}$ ). 
Table 1 Prospectively chosen parameters chosen for inclusion, subdivided into data obtained prospectively from clinical records, and data obtained from questionnaire sent 1 month after diagnosis

\begin{tabular}{|c|c|c|c|c|}
\hline \multirow[b]{2}{*}{ Variable } & \multicolumn{2}{|l|}{ All recruits } & \multicolumn{2}{|c|}{$\begin{array}{l}\text { Respondents to questionnaire sent } \\
1 \text { month after diagnosis }\end{array}$} \\
\hline & All (737) & Missings & All (492) & Missings \\
\hline \multicolumn{5}{|l|}{ Data from clinical records } \\
\hline \multicolumn{5}{|l|}{ Final diagnosis } \\
\hline Miscarriage & $537(73 \%)$ & $0(0 \%)$ & $366(74 \%)$ & $0(0 \%)$ \\
\hline Ectopic & $116(16 \%)$ & $0(0 \%)$ & $75(15 \%)$ & $0(0 \%)$ \\
\hline Resolved PUL & $84(11 \%)$ & $0(0 \%)$ & $51(10 \%)$ & $0(0 \%)$ \\
\hline Age (in years) & $34(5)$ & $0(0 \%)$ & $35(5)$ & $0(0 \%)$ \\
\hline IVF this pregnancy & $50(7 \%)$ & $0(0 \%)$ & $38(8 \%)$ & $0(0 \%)$ \\
\hline Gestation at diagnosis & $65.4(20.0)$ & $130(18 \%)$ & $66.3(19.5)$ & $84(17 \%)$ \\
\hline Nights admission & $0.3(0.7)$ & $12(2 \%)$ & $0.3(0.7)$ & $2(0.4 \%)$ \\
\hline Nights admission (yes vs no) & $163(22 \%)$ & $12(2 \%)$ & $100(20 \%)$ & $2(0.4 \%)$ \\
\hline Days from first scan to diagnosis & $5.0(7.7)$ & $13(2 \%)$ & $5.1(7.4)$ & $6(1 \%)$ \\
\hline \multicolumn{5}{|l|}{ Final management } \\
\hline Medical management & $73(10 \%)$ & $12(2 \%)$ & $45(9 \%)$ & $2(0.4 \%)$ \\
\hline Surgical management & $408(56 \%)$ & $12(2 \%)$ & 291 (59\%) & $2(0.4 \%)$ \\
\hline No treatment needed & $244(34 \%)$ & $12(2 \%)$ & $154(31 \%)$ & $2(0.4 \%)$ \\
\hline \multicolumn{5}{|l|}{ Fetal heart (misc only) } \\
\hline Yes & $126(23 \%)$ & $4(1 \%)$ & $84(23 \%)$ & $3(1 \%)$ \\
\hline No & $407(76 \%)$ & $4(1 \%)$ & $279(77 \%)$ & $3(1 \%)$ \\
\hline \multicolumn{5}{|l|}{ Data from first questionnaire } \\
\hline \multicolumn{5}{|l|}{ Highest level of education } \\
\hline No formal qualifications & $6(1 \%)$ & $233(32 \%)$ & $6(1 \%)$ & $0(0 \%)$ \\
\hline GCSEs (or equivalent) & $41(8 \%)$ & $233(32 \%)$ & $41(8 \%)$ & $0(0 \%)$ \\
\hline A levels (or equivalent) & $52(10 \%)$ & $233(32 \%)$ & $50(10 \%)$ & $0(0 \%)$ \\
\hline Uni degree/prof. qualif. & $278(55 \%)$ & $233(32 \%)$ & $271(55 \%)$ & $0(0 \%)$ \\
\hline Postgraduate/PhD & $127(25 \%)$ & $233(32 \%)$ & $124(25 \%)$ & $0(0 \%)$ \\
\hline \multicolumn{5}{|l|}{ Time taken to conceive } \\
\hline Not planned & $110(22 \%)$ & $240(33 \%)$ & $110(22 \%)$ & $0(0 \%)$ \\
\hline$\leq 1$ year & $305(61 \%)$ & $240(33 \%)$ & $301(61 \%)$ & $0(0 \%)$ \\
\hline$>1$ year & $82(16 \%)$ & $240(33 \%)$ & $81(16 \%)$ & $0(0 \%)$ \\
\hline \multicolumn{5}{|l|}{ Psych disorder } \\
\hline Currently & $21(4 \%)$ & $236(32 \%)$ & $20(4 \%)$ & $3(1 \%)$ \\
\hline In the past & $86(17 \%)$ & $236(32 \%)$ & $83(17 \%)$ & $3(1 \%)$ \\
\hline No & $394(79 \%)$ & $236(32 \%)$ & $386(79 \%)$ & $3(1 \%)$ \\
\hline Any previous pregnancy loss & $264(46 \%)$ & $162(22 \%)$ & $182(37 \%)$ & $0(0 \%)$ \\
\hline Any previous termination & $161(23 \%)$ & $25(3 \%)$ & $121(25 \%)$ & $0(0 \%)$ \\
\hline Any previous children & $316(44 \%)$ & $24(3 \%)$ & $203(41 \%)$ & $0(0 \%)$ \\
\hline \multicolumn{5}{|l|}{ Ethnicity } \\
\hline Asian & 50 & $233(32 \%)$ & 48 & $0(0 \%)$ \\
\hline Black & 53 & $233(32 \%)$ & 51 & $0(0 \%)$ \\
\hline Other & 53 & $233(32 \%)$ & 52 & $0(0 \%)$ \\
\hline White British & 187 & 233 (32\%) & 185 & $0(0 \%)$ \\
\hline White other & 161 & 233 (32\%) & 156 & $0(0 \%)$ \\
\hline
\end{tabular}

GCSE, General Certificate of Secondary Education; IVF, in vitro fertilisation; PUL, pregnancy of unknown location. 


\section{DISCUSSION}

Psychological morbidity (PTS, anxiety or depression) appears to be more common in those with psychiatric history, and in those with a history of pregnancy loss. There is a modest suggestion of potential prognostic ability according to time taken to conceive and ethnicity. Individually, however, all factors have modest AUCs and explain little of the variation in the outcomes (even taking into consideration that $\mathrm{R}^{2}$ values for binary endpoints tend to be modest in general). The most promising factors could be considered for inclusion in a prediction model in future research, but our results indicate that such model may be of limited utility. A considerable proportion of women with psychological morbidity will probably have none of the potentially prognostic factors from our study.

The strength of this study is in its large size relative to other studies in this area, across a diverse population from three central London hospitals, and in the assessment of multiple, prospectively chosen potential prognostic factors. Another strength lies in its inclusion of women with EP, which have not been the subject of any such analysis to date. Only one small study has previously assessed for prognostic factors for PTS, which our group has found to be the most commonly endorsed condition. ${ }^{3}$

A weakness is a considerable drop-out between recruitment and response to the first questionnaire (though unavoidable in studies of this nature, and similar to other studies in this field). It was also necessary to use screening questionnaires rather than the gold-standard of individualised assessment by a trained professional.

A decision was made to assess for factors predictive of psychological morbidity at 1 month rather than at later time points because (1) response rates were expected to be higher at 1 month and (2) this avoids the impact of further pregnancy (healthy or otherwise). However, arguably the most clinically important responses are those that persist over time, and therefore assessing for predictors of longer-term PTS could also be of value.

Previous studies have found higher anxiety and depression in women without children, and with reducing numbers of existing children. ${ }^{5-7}$ This study did not suggest that the absence of children was able to predict those with morbidity. This may be in part due to methodological reasons: for example, this study uses a categorical (presence of psychological morbidity) rather than a continuous outcome (scores from psychometric questionnaires), as a pathological level of symptoms was felt to be the important outcome to try and predict. It is also possible that there have been cultural shifts over the past three decades: modern day women may be more susceptible to distress relating to the loss itself than concern over childlessness. Previous studies have also found that gestation may be associated with increased anxiety, depression and PTS scores. ${ }^{312}$ In contrast to these studies, we did not include stillbirths. Moreover, the vast majority of included women experienced losses in the first 12 weeks of pregnancy (mean gestation 71 days for miscarriage (SD 17), and 46 days (SD 18) for ectopic pregnancies), limiting power to detect differences between the first and second trimester.

In this study, although delay to conception showed a suggestion of modest predictive potential, IVF conception did not. A previous study in Hong Kong suggested IVF pregnancies were associated with higher traumatic impact after loss, ${ }^{8}$ but excluded those with children or with a history of any psychiatric illness, and also used a continuous outcome measure, which may underlie the difference.

Neither gestation nor overnight admission (which is likely to indicate severe pain, heavy blood loss or the need for emergency surgery) seem to be prognostic of psychological distress. This sends an important message to clinicians: even diagnoses at very early gestations (often referred to as 'biochemical pregnancies'), and with clinically mild symptoms, may provoke significant psychological sequelae, and must be treated with compassion.

In 2011, criteria for the diagnosis of miscarriage were changed in order to minimise the possibility of error and inadvertent termination. ${ }^{17}$ As a result, the scan outcome of a 'pregnancy of uncertain viability', which requires a repeat scan 7-14 days later for confirmation, has become

Table 2 AUC, Nagelkerke $\mathrm{R}^{2}$ and $\mathrm{p}$ value in the prediction of any morbidity (anxiety, depression or post-traumatic stress) for each potential prognostic factor, displayed in order of decreasing AUC

\begin{tabular}{|c|c|c|c|}
\hline Predictor & AUC (95\% Cl) & $\mathbf{R}^{2}$ & $P$ value \\
\hline $\begin{array}{l}\text { Psychiatric disorder (no, in past, } \\
\text { currently) }\end{array}$ & 0.61 ( 0.55 to 0.66$)$ & 0.084 & $<0.0001$ \\
\hline $\begin{array}{l}\text { Any previous pregnancy loss } \\
(\mathrm{no}=0 ; \text { yes }=1)\end{array}$ & 0.59 (0.54 to 0.64$)$ & 0.043 & $<0.0001$ \\
\hline $\begin{array}{l}\text { Ethnicity (white British/white } \\
\text { other/black/Asian/other) }\end{array}$ & 0.57 (0.52 to 0.63 ) & 0.024 & 0.07 \\
\hline $\begin{array}{l}\text { Time to conceive } \\
\text { (unknown/s } 1 \text { year/>1 year) }\end{array}$ & $0.56\left(\begin{array}{lll}0.51 & 0.61)\end{array}\right.$ & 0.022 & 0.02 \\
\hline $\begin{array}{l}\text { Final diagnosis (miscarriage, } \\
\text { ectopic, other) }\end{array}$ & 0.54 (0.48 to 0.59$)$ & 0.009 & 0.19 \\
\hline $\begin{array}{l}\text { Final management (surgical, } \\
\text { medical, conservative) }\end{array}$ & 0.54 ( 0.49 to 0.59 ) & 0.008 & 0.23 \\
\hline $\begin{array}{l}\text { Fetal heart (no=0; yes }=1 \text { ) } \\
\text { (miscarriage only) }\end{array}$ & 0.53 (0.47 to 0.59$)$ & 0.008 & 0.34 \\
\hline Age (in years) & 0.53 (0.47 to 0.58$)$ & 0.001 & 0.53 \\
\hline $\begin{array}{l}\text { Educational attainment (none; } \\
\text { GCSE; A-level; university; } \\
\text { postgraduate degree) }\end{array}$ & 0.52 (0.47 to 0.57 ) & 0.003 & 0.89 \\
\hline $\begin{array}{l}\text { Overnight admission }(\mathrm{no}=0 ; \\
\text { yes }=1 \text { ) }\end{array}$ & 0.52 (0.47 to 0.57$)$ & 0.003 & 0.29 \\
\hline $\begin{array}{l}\text { Previous termination }(\mathrm{no}=0 ; \\
\text { yes }=1 \text { ) }\end{array}$ & 0.52 ( 0.47 to 0.57 ) & 0.002 & 0.37 \\
\hline Previous children $(\mathrm{no}=0 ;$ yes $=1$ ) & 0.52 (0.47 to 0.57$)$ & 0.002 & 0.39 \\
\hline $\begin{array}{l}\text { Days to diagnosis from first } \\
\text { scan (days) }\end{array}$ & 0.51 ( 0.46 to 0.57 ) & 0.000 & 0.95 \\
\hline IVF (no=0; yes=1) & 0.50 (0.45 to 0.56$)$ & 0.000 & 0.70 \\
\hline Gestational age (days) & 0.50 (0.44 to 0.56$)$ & 0.000 & 0.89 \\
\hline
\end{tabular}

AUC, area under curve; GCSE, General Certificate of Secondary Education; IVF, in vitro fertilisation. 

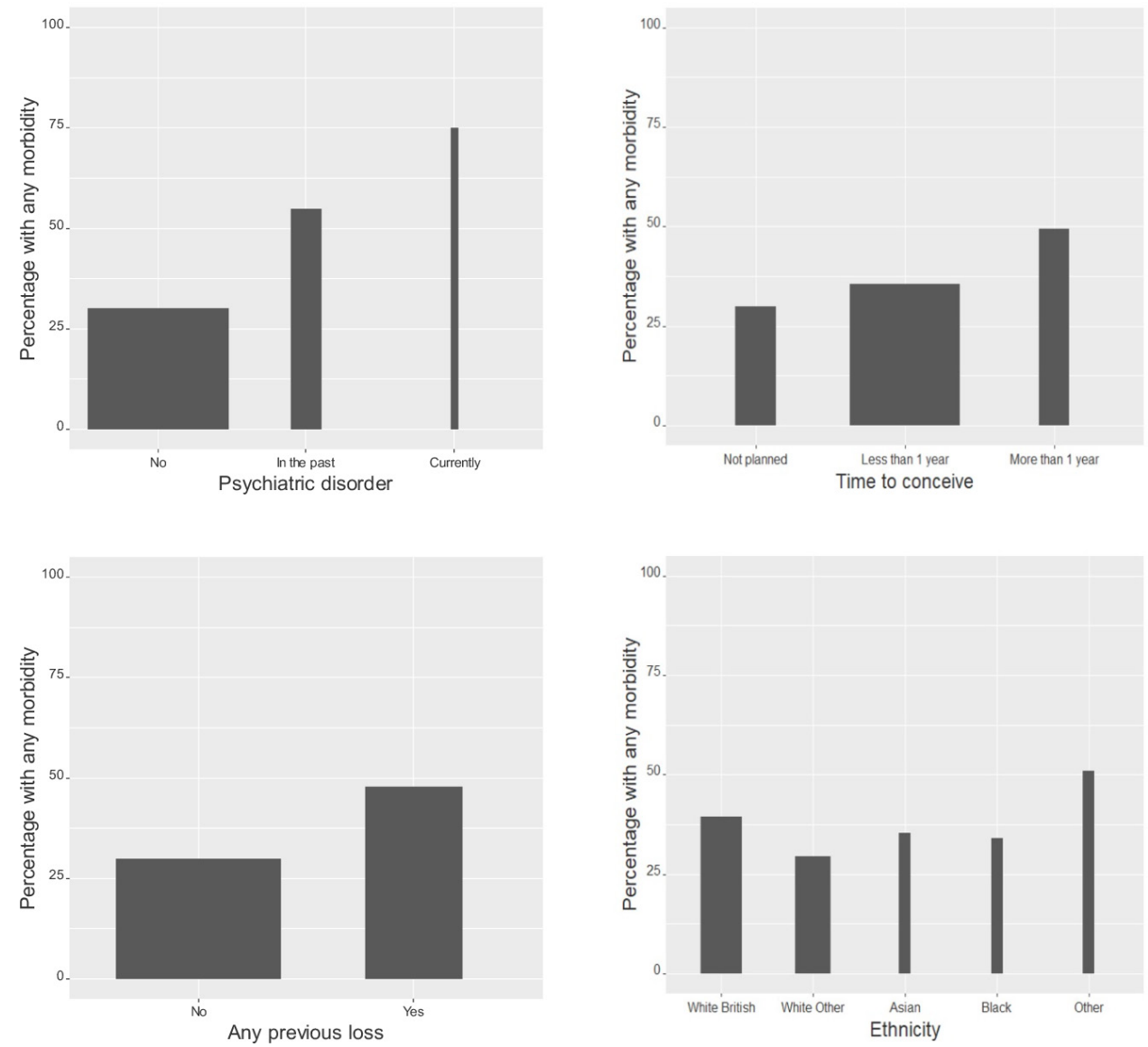

Figure 2 Figures showing presence of any morbidity for the most important prognostic factors. The width of the bars reflects the number of patients with that value.

\begin{tabular}{|c|c|c|c|c|}
\hline & $\begin{array}{l}\text { Anxiety }(\%) \\
(\mathrm{N}=492)\end{array}$ & $\begin{array}{l}\text { Depression (\%) } \\
(\mathrm{N}=492)\end{array}$ & $\begin{array}{l}\text { PTS (\%) } \\
\text { (N=487) }\end{array}$ & $\begin{array}{l}\text { Any (\%) } \\
\text { (N=487) }\end{array}$ \\
\hline \multicolumn{5}{|l|}{ Psych disorder } \\
\hline No & $76 / 386(20 \%)$ & 28/386 (7\%) & $86 / 382(23 \%)$ & $115 / 382(30 \%)$ \\
\hline Yes, in the past & 29/83 (35\%) & 20/83 (24\%) & 39/82 (48\%) & 45/82 (55\%) \\
\hline Yes, currently & $12 / 20(60 \%)$ & $4 / 20(20 \%)$ & $11 / 20(55 \%)$ & 15/20 (75\%) \\
\hline \multicolumn{5}{|l|}{ Any previous loss } \\
\hline No & $59 / 310(19 \%)$ & $20 / 310(6 \%)$ & 73/307 (24\%) & 92/307 (30\%) \\
\hline Yes & 60/182 (33\%) & $33 / 182(18 \%)$ & $66 / 180(37 \%)$ & $86 / 180(48 \%)$ \\
\hline \multicolumn{5}{|l|}{ Ethnicity } \\
\hline Asian & $14 / 48(29 \%)$ & $5 / 48(10 \%)$ & $12 / 48(25 \%)$ & $17 / 48(35 \%)$ \\
\hline White other & 23/156 (15\%) & 10/156 (6\%) & $38 / 156(24 \%)$ & 46/156 (29\%) \\
\hline \multicolumn{5}{|l|}{ Time to conceive } \\
\hline Unknown/unplanned & $20 / 110(18 \%)$ & 9/110 (8\%) & $24 / 110(22 \%)$ & $33 / 110(30 \%)$ \\
\hline$\leq 1$ year & 76/301 (25\%) & $30 / 301(10 \%)$ & $79 / 296(27 \%)$ & 105/296 (35\%) \\
\hline$>1$ year & 23/81 (28\%) & $14 / 81(17 \%)$ & $36 / 81$ (44\%) & 40/81 (49\%) \\
\hline
\end{tabular}

PTS, post-traumatic stress. 
more common. It could be hypothesised that this longer delay to diagnosis (which might also increase the likelihood of unplanned passage of pregnancy tissue outside of the hospital) could have psychological implications: it is reassuring that a longer delay to diagnosis does not seem to be prognostic of morbidity. It is possible that appropriate counselling about the likely outcome, or the increased opportunity for discussion with healthcare professionals during follow-up, might ameliorate any potential negative impact of a delay.

Going forward, it is possible that screening for psychological morbidity after a loss will be a more appropriate way of targeting treatment than a prediction model. The optimal methods and timing of such screening, and its reliability, requires further research.

\section{CONCLUSIONS}

It is unlikely that a useful model to predict psychological distress in the aftermath of EPL can be developed. Clinicians should be particularly alert to the risk of morbidity in those with psychiatric history, and those with previous losses. However, it is imperative that staff working in early pregnancy are vigilant to the risk of disabling mental health conditions in all women after pregnancy loss, irrespective of their gestation, the details of their clinical encounter or their obstetric history.

\section{Author affiliations}

${ }^{1}$ Tommy's National Centre for Miscarriage Research, Queen Charlotte's and Chelsea Hospital, Imperial College, London, UK

${ }^{2}$ Obstetrics and Gynaecology, St Mary's Hospital, London, UK

${ }^{3}$ Department of Development \& Regeneration, KU Leuven, Leuven, Belgium

${ }^{4}$ Obstetrics and Gynaecology, Chelsea and Westminster Hospital, London, UK

${ }^{5}$ Department of Biomedical Data Sciences, Leiden University Medical Center,

Leiden, The Netherlands

${ }^{6}$ Obstetrics and Gynaecology, University Hospitals KU Leuven, Leuven, Belgium

${ }^{7}$ Department of Metabolism, Digestion and Reproduction, Imperial College, London, UK

\section{Twitter Tom Bourne @proftombourne}

Acknowledgements First and foremost, we would also like to thank all women and partners for their participation, especially at an already difficult time. Thanks to Karen Joash for her help originally developing the study protocol and obtaining ethical approval. We would also like to acknowledge Sophie Tapp, Maria Pipi and Alison Perry for their help in recruiting women to this study, and Nenen de la Cruz, Jo Anne McKay, Debbie Gould, Shahla Ahmed, Cecilia Bottomley, Maeve Tuomey and Catriona Stalder for helping the research team to identify potential recruits.

Contributors TB and MJ devised the original study protocol, which was amended by JF. JF, NM-J, ST, SB and MA-M recruited participants for the study. NF and BVC were responsible for statistical analysis of the results. JF, TB and MJ wrote the first draft of the manuscript that was then critically reviewed and revised by the other coauthors. DT commented on the drafts of the paper. All authors approved the final version of the manuscript for submission. All authors had full access to all of the data (including statistical reports and tables) in the study and can take responsibility for the integrity of the data and the accuracy of the data analysis. TB is the guarantor, and affirms that the manuscript is an honest, accurate and transparent account of the study being reported; and that any discrepancies from the study as planned have been explained.

Funding JF was supported by Imperial Health Charity grant number 141517. TB is supported by the National Institute for Health Research (NIHR) Biomedical Research Centre based at Imperial College Healthcare NHS Trust and Imperial College London. The early pregnancy unit at Queen Charlottes and Chelsea Hospital is supported by the Tommy's charity. DT is Senior Clinical Investigator of FWO (Research Foundation-Flanders).

Disclaimer The views expressed are those of the author(s) and not necessarily those of the NHS, the NIHR or the Department of Health.

Competing interests None declared.

Patient consent for publication Not applicable.

Ethics approval This study involves human participants and was approved by NRES committee of South-West Exeter, reference 11/SW/0052 Participants gave informed consent to participate in the study before taking part.

Provenance and peer review Not commissioned; externally peer reviewed.

Data availability statement Data are available upon reasonable request.

Supplemental material This content has been supplied by the author(s). It has not been vetted by BMJ Publishing Group Limited (BMJ) and may not have been peer-reviewed. Any opinions or recommendations discussed are solely those of the author(s) and are not endorsed by BMJ. BMJ disclaims all liability and responsibility arising from any reliance placed on the content. Where the content includes any translated material, BMJ does not warrant the accuracy and reliability of the translations (including but not limited to local regulations, clinical guidelines, terminology, drug names and drug dosages), and is not responsible for any error and/or omissions arising from translation and adaptation or otherwise.

Open access This is an open access article distributed in accordance with the Creative Commons Attribution Non Commercial (CC BY-NC 4.0) license, which permits others to distribute, remix, adapt, build upon this work non-commercially, and license their derivative works on different terms, provided the original work is properly cited, appropriate credit is given, any changes made indicated, and the use is non-commercial. See: http://creativecommons.org/licenses/by-nc/4.0/.

\section{ORCID iDs}

Jessica Farren http://orcid.org/0000-0002-8082-6499

Maria Jalmbrant http://orcid.org/0000-0001-7587-1140

Nora Falconieri http://orcid.org/0000-0002-9533-0859

Nicola Mitchell-Jones http://orcid.org/0000-0003-0299-9586

Shabnam Bobdiwala http://orcid.org/0000-0003-0540-2191

Maya Al-Memar http://orcid.org/0000-0002-3662-3010

Nina Parker http://orcid.org/0000-0002-7962-4451

Ben Van Calster http://orcid.org/0000-0003-1613-7450

Dirk Timmerman http://orcid.org/0000-0002-3707-6645

Tom Bourne http://orcid.org/0000-0003-1421-6059

\section{REFERENCES}

1 Farren J, Jalmbrant M, Falconieri N, et al. Posttraumatic stress, anxiety and depression following miscarriage and ectopic pregnancy: a multicenter, prospective, cohort study. Am J Obstet Gynecol 2020;222:367.e1-367.e22.

2 Cumming GP, Klein S, Bolsover D, et al. The emotional burden of miscarriage for women and their partners: trajectories of anxiety and depression over 13 months. BJOG 2007;114:1138-45.

3 Engelhard IM, van den Hout MA, Arntz A. Posttraumatic stress disorder after pregnancy loss. Gen Hosp Psychiatry 2001;23:62-6.

4 Murphy FA, Lipp A, Powles DL. Follow-Up for improving psychological well being for women after a miscarriage. Cochrane Database Syst Rev 2012;3:CD008679.

5 Neugebauer R, Kline J, O'Connor P, et al. Determinants of depressive symptoms in the early weeks after miscarriage. Am J Public Health 1992;82:1332-9.

6 Thapar AK, Thapar A. Psychological sequelae of miscarriage: a controlled study using the general health questionnaire and the hospital anxiety and depression scale. Br J Gen Pract 1992;42:94-6.

7 Sham Akwan-ho, Yiu Mgar-chung, Ho Wyau-bong. Psychiatric morbidity following miscarriage in Hong Kong. Gen Hosp Psychiatry 2010;32:284-93.

8 Cheung CS, Chan $\mathrm{CH}, \mathrm{Ng} \mathrm{EH}$. Stress and anxiety-depression levels following first-trimester miscarriage: a comparison between women who conceived naturally and women who conceived with assisted reproduction. BJOG 2013;120:1090-7.

9 Friedman T, Gath D. The psychiatric consequences of spontaneous abortion. Br J Psychiatry 1989;155:810-3.

10 Broen AN, Moum T, Bödtker AS, et al. Predictors of anxiety and depression following pregnancy termination: a longitudinal five-year follow-up study. Acta Obstet Gynecol Scand 2006;85:317-23. 
11 Walker TM, Davidson KM. A preliminary investigation of psychological distress following surgical management of early pregnancy loss detected at initial ultrasound scanning: a trauma perspective. J Reprod Infant Psychol 2001;19:7-16.

12 Janssen HJ, Cuisinier MC, Hoogduin KA, et al. Controlled prospective study on the mental health of women following pregnancy loss. Am J Psychiatry 1996;153:226-30.

13 Farren J, Jalmbrant M, Falconieri N, et al. Differences in posttraumatic stress, anxiety and depression following miscarriage or ectopic pregnancy between women and their partners: multicenter prospective cohort study. Ultrasound Obstet Gynecol 2021;57:141-8.
14 Farren J, Jalmbrant M, Ameye L, et al. Post-traumatic stress, anxiety and depression following miscarriage or ectopic pregnancy: a prospective cohort study. BMJ Open 2016;6:e011864.

15 Farren J, Mitchell-Jones N, Verbakel JY, et al. The psychological impact of early pregnancy loss. Hum Reprod Update 2018;24:731-49.

16 Ehring T, Kleim B, Clark DM, et al. Screening for posttraumatic stress disorder: what combination of symptoms predicts best? J Nerv Ment Dis 2007;195:1004-12.

17 NICE. Ectopic pregnancy and miscarriage: diagnosis and initial management, 2012. 